\title{
Performance Analysis of Buffer-Aided Relaying System Based on Data and Energy Coupling Queuing Model for Cooperative Communication Networks
}

\author{
Guangjun Liang, ${ }^{1,2}$ Qi Zhu, ${ }^{1}$ Jianfang Xin,,2 Jiashan Tang, ${ }^{1}$ and Tianjiao Zhang ${ }^{1}$ \\ ${ }^{1}$ College of Telecommunications and Information Engineering, Nanjing University of Posts and Telecommunications, \\ Nanjing 210003, China \\ ${ }^{2}$ Electrical Engineering School, Anhui Polytechnic University, Wuhu 241000, China
}

Correspondence should be addressed to Qi Zhu; zhuqi@njupt.edu.cn

Received 8 November 2016; Accepted 13 March 2017; Published 18 April 2017

Academic Editor: Rafael Pérez-Jiménez

Copyright (C) 2017 Guangjun Liang et al. This is an open access article distributed under the Creative Commons Attribution License, which permits unrestricted use, distribution, and reproduction in any medium, provided the original work is properly cited.

\begin{abstract}
We focus on the performance analysis of the buffer-aided relaying system which allows data and energy packets to arrive independently and depart interactively. First, we profile the cooperative relaying system model as a data arrival and energy arrival coupling queuing model. Considering the influence of channel condition on the data departure rate, a new relay transmit protocol which permits exhausting more energy packet to send one data packet in the bad channel environment is proposed. Second, the joint data packet and energy packet handling problem is ascribed to a Coupled Processor Queuing Model which could achieve its steady state transition probability by Quasi-Birth and Death method. Third, the expressions of throughput, delay, and packet drop rate for both data queue and energy queue are also derived. Simulations are demonstrated to verify the analytical results under different data arrival rate, energy arrival rate, and relaying strategy.
\end{abstract}

\section{Introduction}

Cooperative relaying has fascinating charm in wireless communication networks for boosting system performance parameters significantly in the last decade [1-3]. Recently, a novel style of relay named buffer-aided relay which allows the relays to be equipped with data buffers has attracted significant attention for the purpose of enhancing system throughput, boosting energy efficiency, and improving communication quality $[3,4]$.

The authors in [5] maximize the average throughput performance which can be achieved by simple closed-form formulas. Reference [5] adopts a practical relaying system over a hybrid strategy for three different models, such as halfduplex orthogonal access, full duplex nonorthogonal access, and orthogonal access. In multiple relay scenario with [6, 7], the authors in [6] both propose a new relay section scheme and analyze the performance of the average data packet throughput and delay under several different channel models. Applying Markov chain method in the buffer-aided relaying networks, the authors in [7] model the state transmission matrix of max-link selection strategy and then deduce the closed-form expressions of the outage probability. With a novel multiuser uplink buffer-aided relay protocol presented in [8], the authors analyze the outage probability and delay performance of system and optimize the power allocation in order to minimize the outage probability under the total transmission power constraint. In a word, all in [58] adopt the data buffer-aided relay without considering the energy buffer equipped which can dramatically strengthen the outage performance in relaying networks at the cost of long transmission delay.

Energy harvesting (EH) technique [9] which can collect the energy of ambient environment has recently been deemed to deal with energy constrained issue for the future green wireless communication scenarios. Considering the strategy of both data arrival and energy arrival in [10], an optimal data packet dispatching scheme which can change the transfer rate adaptively to minimize the data packet delay is proposed in the end-to-end $\mathrm{EH}$ wireless communication 
networks. In [11] with classic three-node decode-and-forward (DF) relaying model, the authors adopt $\mathrm{EH}$ source and $\mathrm{EH}$ relay to replace traditional data transmission nodes. A data throughput maximization problem is investigated to obtain the optimal power allocation for delay-constrained traffic and no-delay-constrained traffic, respectively. The authors in [12] address the delay limited throughput maximization problems for an energy harvesting transmitter equipped with finite data buffer and energy buffer in the single user channel, the twoway channel, and the two-way relay channel, respectively. Further, both $[13,14]$ consider the resource optimal problem for the multirelay scenario with limited data and energy buffers. A network throughput maximization problem with a deadline $T$ is formulated as a nonconvex optimization problem which can be solved by the interior-point method in [13]. Then a low-complexity suboptimal scheme at the expense of a slight performance degradation is also proposed. Reference [14] aims at optimizing the long-run average delay of the source packets under the energy neutrality constraint on power consumption of each relay. A distributed power control mechanism for the relaying terminals is developed by using stochastic game method. Specifically, all in [10-14] adopt the buffer-aided relay equipped both data buffer and energy buffer with the assumption of noncausal channel state information (CSI) and energy state information (ESI) which actually are not reasonable in practical scenario.

The authors in [15] propose a harvest-then-transmit (HTT) protocol to jointly optimize the power and time allocation for the purpose of maximizing the system throughput in wireless powered communication network (WPCN). Further, the closed-form expressions of the power and time for the optimization problem are also given. There are two different energy cooperative harvest-and-transmit (HAT) protocols presented for data throughput optimization in [16]. For block-wise HAT protocol, a close-form expression of throughput is achieved. For the block-wise mode adaptation protocol, the authors raise a mode adaptation optimal method to maximize the data throughput of the system. However, all the authors in $[15,16]$ investigate the optimal data transmission with the help of the known EH status which actually dissever the coupling relationship of data arrival and energy arrival. The queue theory [17] is introduced into the cooperative wireless communication scenario to investigate the relaying system performance for jointly considering the data arrival and energy arrival. The authors in $[18,19]$ investigate the throughput, delay, and so forth of system performance parameters by applying queue theory in wireless communication field. Reference [18] focuses on the system performance of Device-to-Device (D2D) communications with variable data arrival and interference. By formulating the queuing model as a Discrete Time Markov Chain (DTMC), the authors adopt model decomposition scheme to iterate out the steady state distribution. A system performance analysis problem is discussed in [19] which considers the data arrival and energy arrival jointly. For the purpose of analyzing the coupling queuing system, the authors present a twostage virtual queuing model which decouples the wait states for the data transmission process depending on the energy arrival process. However, [19] does not consider the influence of channel condition on the coupling relationship of data arrival and energy arrival. In other words, energy exhaust should modify self-adapting with the time varying channel environment.

In this paper, we focus on the performance analysis of data arrival and energy arrival coupling queuing model for cooperative relaying networks. First, we profile the relaying system model with data packet and energy packet independent arrival but interactive departure. Considering the influence of channel condition on the data departure rate, a new relay transmit protocol which allows exhausting more energy packet to send one data packet in the bad channel environment is proposed. Second, the joint data packet and energy packet handling problem is ascribed to a Coupled Processor Queuing Model which could achieve its steady state transition probability by Quasi-Birth and Death method. Third, the expressions of throughput, delay, and packet drop rate for both data queue and energy queue are also derived.

The main contributions of the work are summarized below:

(i) We proposed a new relay adaptive power transmission protocol for data arrival and energy arrival coupling queuing model in cooperative relaying networks. Considering the tradeoff between data delay and energy consumption, we can sacrifice more energy packets to decrease data packet delay when the channel condition is bad and there is sufficient energy.

(ii) Compared with traditional performance analysis of relaying networks, we consider the communication scenario with causal CSI and causal ESI. For practical significance, we expect that data and energy arrival obey Bernoulli distribution or to say that they do not always have data traffic to be sent. With the help of the data buffer and energy buffer, we can take into account the tradeoff between energy consumption and data packet sojourn time to achieve the better system performance in time varying channel surroundings.

(iii) Different from conventional relaying system, we jointly investigate the relaying node with independent to arrive but interactive to depart data packet and energy packet. The cooperative relaying system model which allows data arrival and energy arrival coupling queuing is profiled as a Coupled Processor Queuing Model. Applying the method of Quasi-Birth and Death Process, we can derive the expressions of system performance parameters such as throughput, delay, and packet drop rate for both data queue and energy queue. Some conclusions which can strengthen the system performance with the cost of redundant energy expenditure are discussed.

The remainder of this paper is organized as follows. In Section 2, the system model with a new relay protocol and the queue model are described. In Section 3, the Coupled Processor Queuing Model is solved by a Quasi-Birth and Death method. In Section 4, the performance parameters of throughput, delay, and packet drop rate for both data queue 

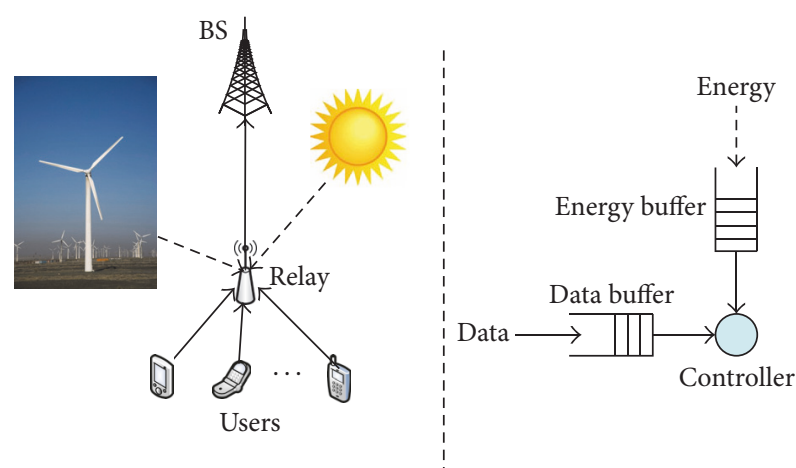

$\begin{aligned} \longrightarrow & \text { Data link } \\ --\rightarrow & \text { Energy link }\end{aligned}$

$\longrightarrow$ Data link

$-\rightarrow$ Energy link

(b)

FIGURE 1: Cellular wireless networks with buffer-aided relaying system; (a) the scenario of data packet and energy packet independent arrival but interactive departure; (b) the queuing model of (a).

and energy queue will be analyzed. In Section 5, both the analysis and the simulation results are proposed to compare the theoretical value and the simulated value. Section 6 give a conclusion for this paper.

\section{System Model and Queue Model}

In this section, we first introduce the adopted system model and the performance measure. A new relay transmit protocol for data arrival and energy arrival coupling queuing model in a cooperative relaying networks is also proposed. Then, the coupling queuing model is profiled.

2.1. System Model. In this paper, we focus on the performance analysis in such a scenario including one source, one EH buffer-aided relay, and $K$ users. Considering EH bufferaided relay multiusers cooperative communication system as in Figure 1(a), all the users transfer the information to the source with the help of the EH relay in the uplink due to shadow effect and fringe effect which generates the users-destination channel too weak to support the direct transmission. Compared with traditional buffer-aided relay [5-8], the EH buffer-aided relay equips two buffers which are one data buffer and one energy buffer. We take into account EH relay technology which makes the best of the energy harvested to transmit the data of cellular users' collaboratively and thus boost the performance of network without additional energy consumption. Specifically, it is not convenient for direct current supply because the relay is equipped in outdoors. So we import a EH relay which relies on energy harvesting totally without continuous supply of power into cellular network. In order to simplify the scenario, we assume that the relay does not consume energy when it receives and processes information from users and source.

The available overall frequency spectrum bandwidth is $W$ which can been divided into $N_{c}$ equal-bandwidth orthogonal channels. Without loss of generality, we assume that all the channels occupy the same bandwidth and experience independent frequency selective fading. Different from the assumption in $[10,11]$, we only need the statistical property of the channel state and energy harvesting state for the purpose of the performance analysis of relaying system. We adopt half-duplex relay protocol which has two equal time slots denoted as $T_{0}$ in a data packet and energy packet processing procedure. The EH style of relay is the same as [15] which first presents a new solution to provide convenient and perpetual energy supplies to wireless networks named as Simultaneous Wireless Information and Power Transfer (SWIPT) energy harvesting.

Compared with traditional relay transmit protocol with Constant Power Transmission (CPT) strategy, the proposed new relay transmit protocol adopts an adaptive power transmission (APT) strategy which can strengthen the system performance with the cost of redundant energy expenditure. The whole transmission cycle is divided into two time slots for the new relay protocol. In the first time slot, each user transmits its data packet to relay if the data buffer of user is not empty. In particular cases there are maybe several users transferring their data packet to relay during the $T_{0}$; for convenience we only allow the relay to receive one data packet which is the first to be sent and the others must resend in the next transmission cycle. Meanwhile the relay also harvests surrounding energy such as solar power, wind power, and other renewable energy sources from ambient environment if corresponding $\mathrm{EH}$ equipment could be equipped. In the second time slot, relay decides to deal with data packets and energy packets according to forward rule beforehand, CSI currently, data and energy buffer state, which means that it will expend one or more energy packets to send one data packet. The unit energy $E_{0}$ which depends on the transfer power and transmission cycle is defined later.

Traditional wireless communication system model [15, 16] assumes that data packet arrives in every continuous time slot which actually is not unreasonable. Different from that, we consider that the data arrival is discontinuous which is modeled as a Bernoulli process with parameter is $p$. We can also assume that energy arrival is modeled as Bernoulli process with parameter is $q$ homologous. For convenience, we stipulate that all the data packets transmitted in the cooperative communication system are the same size denoted as $L_{0}$ with unit interval $T_{0}$ which can be configurable and less than the length of one time slot. We also stipulate that the one or more energy packets will be exhausted when the relay transfers a data packet in a transmission cycle $T_{0}$.

Because the EH process is a successive process which is not convenient to profile, we profile the $\mathrm{EH}$ process as a discrete process with unit energy $E_{0}$ arrival which would be modeled as a Bernoulli process. We also assume that the relay can adaptively change its transmitting power according to the current data and energy buffers' state. Considering the energy packet consumption and data packet transmission, the Shannon capacity with the channel case $i$ in the base station is expressed as

$$
R_{i}=\frac{W}{2 N_{c}} \log \left(1+\frac{P_{i}^{r}\left|h_{i}^{\mathrm{rd}}\right|^{2}}{N_{0}}\right), \quad i=1,2, \ldots, M,
$$


where $P_{i}^{r}$ and $h_{i}^{\text {rd }}$ are relay transmission power and instantaneous channel gain from relay to destination in channel case $i$. The Additive Gaussian White Noise (AWGN) at the base station is zero mean with variances $N_{0}$. With the same assumption in [5-7], the channel is block fading which means that the channel gain from relay to destination in the second time slot remains unchanged. Thus, the relay can transmit one data packet at a constant power $P_{i}^{r}$ in the time length $T_{0}$ with channel case $i$ which means that channel conditions are satisfied using $i$ energy packets to send one data packet. Therefore, we provide the division rule of channel case $i$ as follows:

Channel Case 0. The average channel gain $h_{\text {ave }}^{\text {rd }}$ of current transmission time slot satisfies $h_{\mathrm{ave}}^{\mathrm{rd}} \leq h_{M}^{\mathrm{TH}}$ which means the channel condition is too bad to transmit the data packet.

Channel Case 1. The average channel gain $h_{\text {ave }}^{\text {rd }}$ of current transmission time slot satisfies $h_{\text {ave }}^{\text {rd }} \geq h_{1}^{\text {TH }}$ which means the channel condition is very good to use one energy packet transmit one data packet.

Channel Case 2. The average channel gain $h_{\mathrm{ave}}^{\text {rd }}$ of current transmission time slot satisfies $h_{2}^{\mathrm{TH}} \leq h_{\mathrm{ave}}^{\mathrm{rd}}<h_{1}^{\mathrm{TH}}$ which means the channel condition needs using two energy packet transmit one data packet.

Channel Case $i$. The average channel gain $h_{\mathrm{ave}}^{\text {rd }}$ of current transmission time slot satisfies $h_{i}^{\mathrm{TH}} \leq h_{\mathrm{ave}}^{\mathrm{rd}}<h_{i-1}^{\mathrm{TH}}$ which means the channel condition needs using $i$ energy packet transmit one data packet.

Channel Case $M$. The average channel gain $h_{\mathrm{ave}}^{\mathrm{rd}}$ of current transmission time slot satisfies $h_{M}^{\mathrm{TH}} \leq h_{\mathrm{ave}}^{\text {rd }}<h_{M-1}^{\mathrm{TH}}$ which means the channel condition needs using $M$ energy packet transmit one data packet.

However, if the average channel gain $h_{\text {ave }}^{\text {rd }}$ of current transmission time slot satisfies channel case $i, i=1,2, \ldots, M$ did not mean the data packet will be sent unless the data buffer is nonempty and the energy packet in buffer is no less than $i$.

In the relay APT protocol, the numerical value of $P_{1}^{r}$, $h_{1}^{\mathrm{TH}}$, and $M$ will be installed. The more you set the $M$, the lower the threshold of allowing transmission data packet will get in the worse channel condition. In other words, setting the bigger $M$ value means that we will sacrifice more energy packets to send one data packet. Although we expend even more energy, the better delay will be obtained. There is a tradeoff between energy consumption and delay which will be discussed particularly in the latter simulation section. Because in one data packet transmission process we maintain a constant transmission power $P_{i}^{\text {tr }}$ with time $T_{0}$, the relationship of transmission power in different channel conditions is

$$
P_{1}^{\mathrm{tr}}=\frac{1}{2} P_{2}^{\mathrm{tr}}=\cdots=\frac{1}{i} P_{i}^{\mathrm{tr}}=\cdots=\frac{1}{M} P_{M}^{\mathrm{tr}} .
$$

The corresponding channel condition threshold is deduced by Shannon equation as follows:

$$
\left|h_{1}^{\mathrm{TH}}\right|^{2}=i\left|h_{i}^{\mathrm{TH}}\right|^{2}, \quad i=2,3, \ldots, M .
$$

According to the corresponding channel condition threshold in (3) and relay APT protocol, we can adopt channel statistical property to achieve the probability of in different channel case which is denoted as $p_{m}, m=0,1, \ldots, M$.

2.2. Queue Model. Assuming the same with [10, 11], we stipulate a deterministic data arrival and energy arrival model which actually assures that the condition of data packet and energy packet arrival are known prior to transmission. Different from traditional queue model, in this paper the joint data packet and energy packet handling problem is modeled as a Coupled Processor Queuing Model (CPQM) [20] illustrated in Figure 2(b), where there are one data buffer, one energy buffer, and one single server which represents the buffer-aided relay. For definiteness and without loss of generality, we set the max buffer dimension of data buffer and energy buffer as $N_{d}$ and $N_{e}$. A two-state Markov process $(i, j), i \in\left\{0,1,2, \ldots, N_{d}\right\}, j \in\left\{0,1,2, \ldots, N_{e}\right\}$ is defined to describe the CPQM where $i$ and $j$ denote the data queue length and energy queue length, respectively. For each data queue length $i$, data packet with unit size $L_{0}$ arrives according to Bernoulli process with parameter $p$. Similarly, For each energy queue $j$, energy packet with unit size $E_{0}$ arrives according to Bernoulli process with parameter $q$. The unit data packet size $L_{0}$ and unit energy packet size $E_{0}$ are given as follows:

$$
\begin{aligned}
& L_{0}=\frac{W T_{0}}{2 N_{0}} \log \left(1+\frac{P_{i}^{r}\left|h_{i}^{\mathrm{rd}}\right|^{2}}{N_{0}}\right), \quad i=1,2, \ldots, M, \\
& E_{0}=P_{1}^{\operatorname{tr}} T_{0}=\frac{1}{2} P_{2}^{\operatorname{tr}} T_{0}=\cdots=\frac{1}{i} P_{i}^{\operatorname{tr}} T_{0}=\cdots=\frac{1}{M} P_{M}^{\operatorname{tr}} T_{0} .
\end{aligned}
$$

For the two-dimension Markov process $(i, j)$ where there are $\left(N_{d}+1\right)^{2}\left(N_{e}+1\right)^{2}$ possible states which will be more complicated with the increasing of max buffer dimension $N_{d}$ and $N_{e}$, we can adopt Quasi-Birth and Death (QBD) method to solve it step by step.

\section{QBD Method for Solving CPQM}

In this section, we first redistrict the state space of twodimension Markov process of the data arrival and energy arrival coupling queuing model. Then, the submatrix of transition probability is defined and solved, respectively.

3.1. Redistrict the State Space of CPQM. Firstly, we redistrict the state space of two-dimension Markov process $(i, j), i \in$ $\left\{0,1,2, \ldots, N_{d}\right\}, j \in\left\{0,1,2, \ldots, N_{e}\right\}$ and descript the twodimension static with one-dimension process which will reduce the solving complexity of GBD method. If we define state subset $L_{i}=\left\{(i, j) \mid 0 \leq j \leq N_{e}\right\}, 0 \leq i \leq N_{d}$ where $i$ 


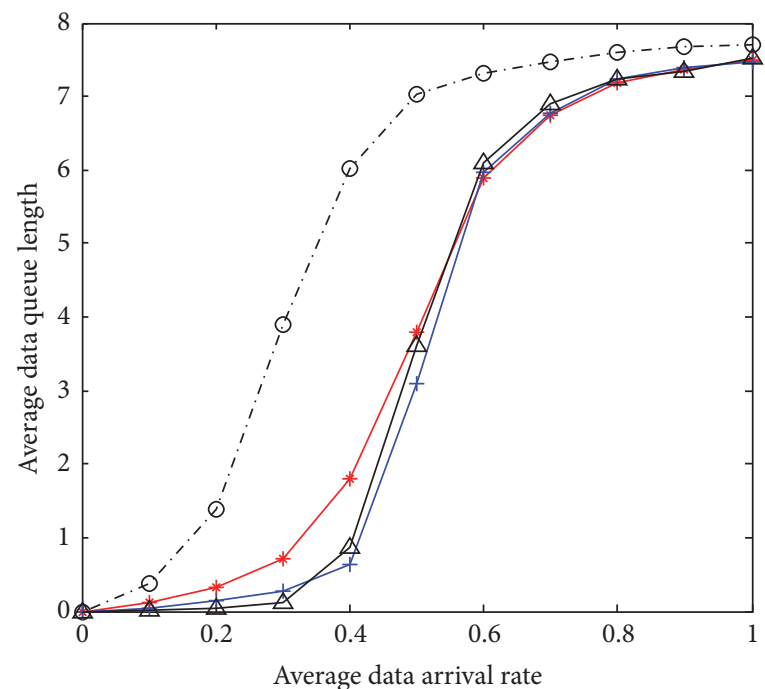

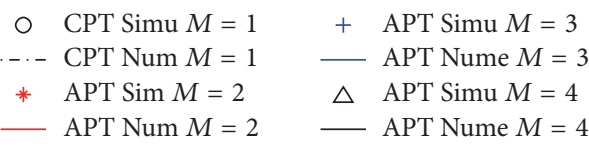

(a) Average data queue length with $q=0.8$

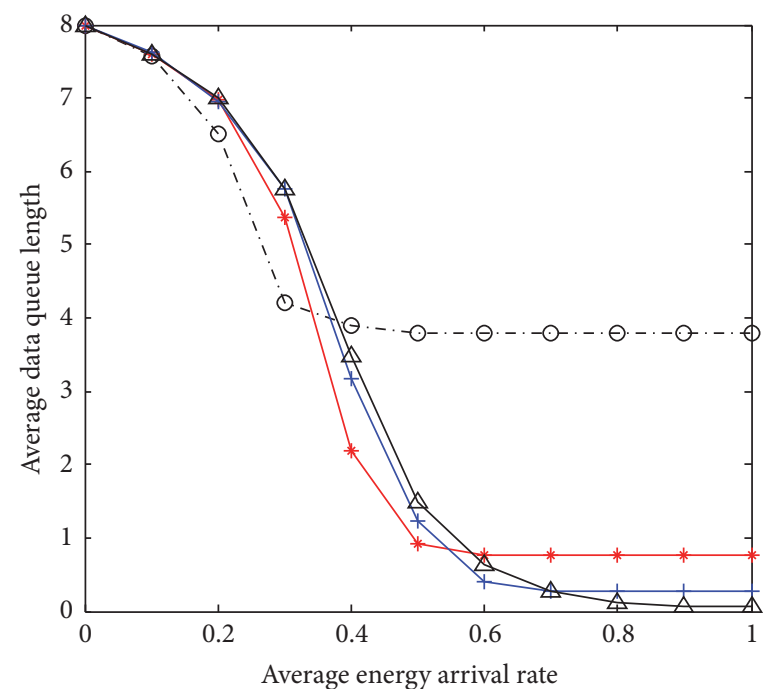

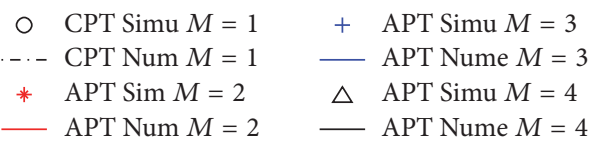

(b) Average data queue length with $p=0.3$

FIgURE 2: The average data queue length of CPQM when $M=1,2,3,4$.

and $j$ are called horizontal and phase, the matrix of transition probability of GBD method is denoted as

$$
\mathbf{P}=\left[\begin{array}{cccccc}
B_{0} & A_{0} & 0 & \cdots & \cdots & 0 \\
C_{1} & B_{1} & A_{1} & \ddots & \ddots & \vdots \\
0 & C_{2} & B_{2} & A_{2} & \ddots & \vdots \\
\vdots & \ddots & \ddots & \ddots & \ddots & 0 \\
\vdots & \ddots & \ddots & \ddots & \ddots & \ddots \\
0 & \cdots & \cdots & 0 & C_{N_{d}} & B_{N_{d}}
\end{array}\right],
$$

where $A_{i}, i=0,1, \ldots, N_{d}-1$ is the submatrix of transition probability which signifies that state subset $L_{i}$ transits to state subset $L_{i+1}$. Also, $B_{i}, i=0,1, \ldots, N_{d}$ is the submatrix of transition probability which signifies that state subset $L_{i}$ transits to state subset $L_{i}$. $C_{i}, i=1,2, \ldots, N_{d}$ is the submatrix of transition probability which signifies that state subset $L_{i}$ transits to state subset $L_{i-1}$.

In order to decide the departure with data packet and the energy packet coupled, we should consider the four possible arrivals conditions which are defined as follows:

$$
\begin{aligned}
& p_{a}=(1-p) *(1-q), \\
& p_{b}=p *(1-q),
\end{aligned}
$$

$$
\begin{aligned}
& p_{c}=(1-p) * q, \\
& p_{d}=p * q,
\end{aligned}
$$

where $p_{a}$ manifest that there is no data packet and energy arrival in the current time slot. Similarly, $p_{b}$ manifest that there is one data packet arrival and no energy arrival in the current time slot. $p_{c}$ manifest that there is no data packet arrival and one energy arrival in the current time slot. $p_{d}$ manifest that there is one data packet arrival and one energy arrival in the current time slot.

3.2. Solving the Submatrix of Transition Probability. The submatrix of transition probability $A_{i}, i=0,1, \ldots, N_{d}-1$, is defined as

$$
A_{i}=\left[\begin{array}{ccccc}
P_{0,0}^{A_{i}} & P_{0,1}^{A_{i}} & 0 & \ldots & 0 \\
0 & P_{1,1}^{A_{i}} & P_{1,2}^{A_{i}} & \ddots & \vdots \\
\vdots & \ddots & \ddots & \ddots & 0 \\
\vdots & \ddots & \ddots & P_{N_{d}-1, N_{d}-1}^{A_{i}} & P_{N_{d}-1, N_{d}}^{A_{i}} \\
0 & \ldots & \ldots & 0 & P_{N_{d}, N_{d}}^{A_{i}}
\end{array}\right],
$$

where $P_{m, n}^{A_{i}}$ with $m, n=0,1, \ldots, N_{d}$ denote the transition probability from state subset $L_{i}$ to state subset $L_{i+1}$. After 
a careful calculation, we find that the elements in the principal diagonal and the $P_{m, n}^{A_{i}}$ with the relationship $m+1=n$ are nonzero value, and the other elements are zero. Joint considering the relay APT protocol with the four possible arrivals condition in formula (6), the nonzero elements in $A_{i}, i=0,1, \ldots, N_{d}-1$ are given as

$$
\begin{aligned}
& P_{j, j}^{A_{i}} \\
& \quad= \begin{cases}p_{b}, & j=0 \\
p_{b} *\left(1-\sum_{n=1}^{j} p_{n}\right), & j=1,2, \ldots, M-1 \\
p_{b} *\left(1-\sum_{n=1}^{M} p_{n}\right), & j=M, M+1, \ldots, N_{e}-1 \\
p * p_{0}, & j=N_{e},\end{cases}
\end{aligned}
$$

$$
\begin{aligned}
& P_{j, j+1}^{A_{i}} \\
& = \begin{cases}p_{d} *\left(1-\sum_{n=1}^{j+1} p_{n}\right), & j=0,1, \ldots, M-2 \\
p_{d} *\left(1-\sum_{n=1}^{M} p_{n}\right), & j=M-1, M, \ldots, N_{e}-1,\end{cases}
\end{aligned}
$$

where $P_{j, j}^{A_{i}}$ is the expression of the principal diagonal element in $A_{i} . P_{j, j+1}^{A_{i}}$ is the expression of the element with the relationship $m+1=n$ in $A_{i}$.

More complicated than $A_{i}$, the submatrix of transition probability $B_{i}, i=0,1, \ldots, N_{d}$ is divided into three parts $B_{0}$, $B_{i}, i=1,2, \ldots, N_{d}-1$, and $B_{N_{d}}$ which will be discussed, respectively. First, the submatrix of transition probability $B_{0}$, is defined as

$$
B_{0}=\left[\begin{array}{cccccccc}
P_{0,0}^{B_{0}} & P_{0,1}^{B_{0}} & 0 & \ldots & \ldots & \cdots & \cdots & 0 \\
P_{1,0}^{B_{0}} & P_{1,1}^{B_{0}} & P_{1,2}^{B_{0}} & \ddots & \ddots & \ddots & \ddots & \vdots \\
\vdots & P_{2,1}^{B_{0}} & P_{2,2}^{B_{0}} & P_{2,3}^{B_{0}} & \ddots & \ddots & \ddots & \vdots \\
P_{M-1,0}^{B_{0}} & \ddots & \ddots & \ddots & \ddots & \ddots & \ddots & \vdots \\
P_{M, 0}^{B_{0}} & P_{M, 1}^{B_{0}} & \ddots & \ddots & \ddots & \ddots & \ddots & \vdots \\
0 & P_{M+1,1}^{B_{0}} & \ddots & \ddots & \ddots & \ddots & \ddots & 0 \\
\vdots & \ddots & \ddots & \ddots & \ddots & P_{N_{d}-1, N_{d}-2}^{B_{0}} & P_{N_{d}-1, N_{d}-1}^{B_{0}} & P_{N_{d}-1, N_{d}}^{B_{0}} \\
0 & \ldots & 0 & P_{N_{d}, N_{d}-M}^{B_{0}} & P_{N_{d}, N_{d}-M+1}^{B_{0}} & \cdots & P_{N_{d}, N_{d}-1}^{B_{0}} & P_{N_{d}, N_{d}}^{B_{0}}
\end{array}\right],
$$

where $P_{m, n}^{B_{0}}$ with $m, n=0,1, \ldots, N_{d}$ denote the transition probability from state subset $L_{i}$ to state subset $L_{i}$. More complicated than $A_{i}$, the $P_{m, n}^{B_{0}}$ with the relationship $m-k=$ $n, k=-1,0,1, \ldots, M$ are nonzero value, and the other elements are zero. Actually, the $P_{m, n}^{B_{0}}$ are the elements in the principal diagonal when $m=n$ or other $k=0$. We jointly consider the relay APT protocol and the four possible arrivals condition in formula (6), and the nonzero elements in the $B_{0}$ are given as

$$
\begin{aligned}
& P_{j, j+1}^{B_{0}}=p_{c}, \quad j=0,1,2, \ldots, N_{e}-1, \\
& P_{j, j}^{B_{0}}= \begin{cases}p_{a}+p_{d} * p_{1}, & j=0,1,2, \ldots, N_{e}-1 \\
1-p, & j=N_{e},\end{cases}
\end{aligned}
$$

$$
\begin{aligned}
& P_{j, j-m}^{B_{0}},\left\{\begin{array}{lr}
p_{b} * p_{m}+p_{d} * p_{m+1}, & j=m, m+1, \ldots, N_{e}-1 \\
p * p_{m}, & j=N_{e},
\end{array}\right. \\
& P_{j, j-m}^{B_{0}}= \begin{cases}p_{b} * p_{m}, & j=m, m+1, \ldots, N_{e}-1 \\
p * p_{m}, & j=N_{e},\end{cases}
\end{aligned}
$$$$
m=M \text {, }
$$

where $P_{j, j+1}^{B_{0}}$ are the expression of the elements with the relationship $m+1=n$ in the submatrix of transition probability $B_{0} . P_{j, j}^{B_{0}}$ are the expression of the principal diagonal element in the $B_{0}$. Also, formulas (12) and (13) are the expression of the 
element with the relationships $m-k=n, k=1,2, \ldots, M-1$, and $m-M=n$ in $B_{0}$, respectively.

$$
B_{i}=\left[\begin{array}{cccccccc}
P_{0,0}^{B_{i}} & P_{0,1}^{B_{i}} & 0 & \ldots & \ldots & \ldots & \ldots & 0 \\
P_{1,0}^{B_{i}} & P_{1,1}^{B_{i}} & P_{1,2}^{B_{i}} & \ddots & \ddots & \ddots & \ddots & \vdots \\
\vdots & P_{2,1}^{B_{i}} & P_{2,2}^{B_{i}} & \ddots & \ddots & \ddots & \ddots & \vdots \\
P_{M-1,0}^{B_{i}} & \ddots & \ddots & \ddots & P_{M-1, M}^{B_{i}} & \ddots & \ddots & \vdots \\
P_{M, 0}^{B_{i}} & \ddots & \ddots & \ddots & P_{M, M}^{B_{i}} & \ddots & \ddots & \vdots \\
0 & \ddots & \ddots & \ddots & \ddots & \ddots & \ddots & 0 \\
\vdots & \ddots & \ddots & \ddots & \ddots & \ddots & P_{N_{d}-1, N_{d}-1}^{B_{i}} & P_{N_{d}-1, N_{d}}^{B_{i}} \\
0 & \ldots & \ddots & P_{N_{d}, N_{d}-M}^{B_{i}} & P_{N_{d}, N_{d}-M+1}^{B_{i}} & \ldots & P_{N_{d}, N_{d}-1}^{B_{i}} & P_{N_{d}, N_{d}}^{B_{i}}
\end{array}\right], \quad i=1,2, \ldots, N_{d}-1,
$$

where $P_{m, n}^{B_{i}}$ with $m, n=0,1, \ldots, N_{d}$ denote the transition probability from state subset $L_{i}$ to state subset $L_{i}$. The same as with $B_{0}$, the $P_{m, n}^{B_{i}}$ with the relationship $m-k=n, k=$ $-1,0,1, \ldots, M$ are nonzero value, and the other elements are zero. Considering the relay APT protocol and the four possible arrivals condition in formula (6) jointly, we can obtain the nonzero elements in the $B_{i}$ given as

$$
P_{j, j+1}^{B_{i}}= \begin{cases}p_{c} *\left(1-\sum_{n=1}^{j} p_{n}\right), & j=0,1, \ldots, M-1 \\ p_{c} *\left(1-\sum_{n=1}^{M} p_{n}\right), & j=M, M+1, \ldots, N_{e},\end{cases}
$$

$P_{j, j}^{B_{i}}$

$$
= \begin{cases}p_{a}+p_{d} * p_{1}, & j=0 \\ p_{a} *\left(1-\sum_{n=1}^{j} p_{n}\right)+p_{d} * p_{1}, & j=1,2, \ldots, M-1 \\ p_{a} *\left(1-\sum_{n=1}^{M} p_{n}\right)+p_{d} * p_{1}, & j=M, M+1, \ldots, N_{e}-1 \\ (1-p) * p_{0}, & j=N_{e},\end{cases}
$$$$
P_{j, j-m}^{B_{i}}= \begin{cases}p_{b} * p_{m}+p_{d} * p_{m+1}, & j=m, m+1, \ldots, N_{e}-1 \\ p * p_{m}, & j=N_{e},\end{cases}
$$

$$
m=1,2, \ldots, M-1,
$$

$$
P_{j, j-m}^{B_{i}}=\left\{\begin{array}{ll}
p_{b} * p_{m}, & j=m, m+1, \ldots, N_{e}-1 \\
p * p_{m}, & j=N_{e},
\end{array} \quad m=M\right.
$$

where $P_{j, j+1}^{B_{i}}$ are the expression of the elements with the relationship $m+1=n$ in the submatrix of transition probability $B_{i} \cdot P_{j, j}^{B_{i}}$ are the expression of the principal diagonal element in the $B_{i}$. Also, formulas (17) and (18) are the expression of the element with the relationships $m-k=$ $n, k=1,2, \ldots, M-1$, and $m-M=n$ in $B_{i}$, respectively.
Second, the submatrix of transition probability $B_{i}, i=$

The third part of submatrix of transition probability $B_{N}$, is defined as

$$
B_{N_{d}}=\left[\begin{array}{ccccc}
P_{0,0}^{B_{N_{d}}} & P_{0,1}^{B_{N_{d}}} & 0 & \cdots & 0 \\
0 & P_{1,1}^{B_{N_{d}}} & P_{1,2}^{B_{N_{d}}} & \ddots & \vdots \\
\vdots & \ddots & \ddots & \ddots & 0 \\
\vdots & \ddots & \ddots & P_{N_{d}-1, N_{d}-1}^{B_{N_{d}}} & P_{N_{d}-1, N_{d}}^{B_{N_{d}}} \\
0 & \cdots & \ldots & 0 & P_{N_{d}, N_{d}}^{B_{N_{d}}}
\end{array}\right]
$$

where $P_{m, n}^{B_{N_{d}}}$ with $m, n=0,1, \ldots, N_{d}$ denote the transition probability from state subset $L_{i}$ to state subset $L_{i}$. The same as with $A_{i}$, the elements in the principal diagonal and the $P_{m, n}^{B_{N_{d}}}$ with the relationship $m+1=n$ are nonzero value, and the other elements are zero. Jointly considering the relay APT protocol and the four possible arrivals condition in formula (6), the nonzero elements in $B_{N_{d}}$ are given as

$$
\begin{aligned}
& P_{j, j}^{B_{N_{d}}} \\
& = \begin{cases}1-q, & j=0 \\
(1-q) *\left(1-\sum_{n=1}^{j} p_{n}\right), & j=1,2, \ldots, M-1 \\
(1-q) *\left(1-\sum_{n=1}^{M} p_{n}\right), & j=M, M+1, \ldots, N_{e}-1 \\
p_{0}, & j=N_{e},\end{cases} \\
& P_{j, j+1}^{B_{N_{d}}} \\
& = \begin{cases}q *\left(1-\sum_{n=1}^{j+1} p_{n}\right), & j=0,1, \ldots, M-2 \\
q *\left(1-\sum_{n=1}^{M} p_{n}\right), & j=M-1, M, \ldots, N_{e}-1,\end{cases}
\end{aligned}
$$

where $P_{j, j}^{B_{N_{d}}}$ and $P_{j, j+1}^{B_{N_{d}}}$ are the expression of the principal diagonal element and the element with the relationship $m+$ $1=n$ in $B_{N_{d}}$, respectively. $1,2, \ldots, N_{d}-1$, is defined as 
The same as with $B_{i}, i=0,1, \ldots, N_{d}$, the submatrix of transition probability $C_{i}, i=1,2, \ldots, N_{d}$ is divided into two parts $C_{i}, i=1,2, \ldots, N_{d}-1$ and $C_{N_{d}}$ which will be deduced in the same way. The submatrix of transition probability $C_{i}, i=$ $1,2, \ldots, N_{d}-1$ and the nonzero elements in them are given as follows:

$$
\begin{aligned}
& C_{i}=\left[\begin{array}{cccccccc}
P_{0,0}^{C_{i}} & 0 & \ddots & \ldots & \ldots & \ldots & \ldots & 0 \\
P_{1,0}^{C_{i}} & P_{1,1}^{C_{i}} & \ddots & \ddots & \ddots & \ddots & \ddots & \vdots \\
\vdots & P_{2,1}^{C_{i}} & P_{2,2}^{C_{i}} & \ddots & \ddots & \ddots & \ddots & \vdots \\
P_{M-1,0}^{C_{i}} & \ddots & \ddots & \ddots & \ddots & \ddots & \ddots & \vdots \\
P_{M, 0}^{C_{i}} & \ddots & \ddots & \ddots & \ddots & \ddots & \ddots & \vdots \\
0 & \ddots & \ddots & \ddots & \ddots & \ddots & \ddots & \vdots \\
\vdots & \ddots & \ddots & \ddots & \ddots & P_{N_{d}-1, N_{d}-2}^{C_{i}} P_{N_{d}-1, N_{d}-1}^{C_{C}} & 0 \\
0 & \cdots & 0 & P_{N_{d}, N_{d}-M}^{C_{i}} P_{N_{d}, N_{d}-M+1}^{C_{i}} & \ldots & P_{N_{d}, N_{d}-1}^{C_{i}} & P_{N_{d}, N_{d}}^{C_{i}}
\end{array}\right], \quad i=1,2, \ldots, N_{d}-1, \\
& P_{j, j}^{C_{i}}= \begin{cases}p_{c} * p_{1}, & j=1,2, \ldots, N_{e}-1 \\
0, & j=N_{e}\end{cases} \\
& P_{j, j-m}^{C_{i}}=\left\{\begin{array}{ll}
p_{a} * p_{m}+p_{c} * p_{m+1}, & j=m, m+1, \ldots, N_{e}-1 \\
(1-p) * p_{m}, & j=N_{e},
\end{array} \quad m=1,2, \ldots, M-1,\right. \\
& P_{j, j-m}^{C_{i}}=\left\{\begin{array}{ll}
p_{a} * p_{m}, & j=m, m+1, \ldots, N_{e}-1 \\
(1-p) * p_{m}, & j=N_{e},
\end{array} \quad m=M .\right.
\end{aligned}
$$

We can adopt the same method to acquire the submatrix of transition probability $C_{N_{d}}$ and the nonzero elements in them are given as follows:

$$
\begin{aligned}
& C_{N_{d}}=\left[\begin{array}{cccccccc}
P_{0,0}^{C_{N_{d}}} & 0 & \ldots & \ldots & \ldots & \ldots & \ldots & 0 \\
P_{1,0}^{C_{N_{d}}} & P_{1,1}^{C_{N_{d}}} & \ddots & \ddots & \ddots & \ddots & \ddots & \vdots \\
\vdots & P_{2,1}^{C_{N_{d}}} & P_{2,2}^{C_{N_{d}}} & \ddots & \ddots & \ddots & \ddots & \vdots \\
P_{M-1,0}^{C_{N_{d}}} & \ddots & \ddots & \ddots & \ddots & \ddots & \ddots & \vdots \\
P_{M, 0}^{C_{N_{d}}} & \ddots & \ddots & \ddots & \ddots & \ddots & \ddots & \vdots \\
0 & \ddots & \ddots & \ddots & \ddots & \ddots & \ddots & \vdots \\
\vdots & \ddots & \ddots & \ddots & \ddots & P_{N_{d}-1, N_{d}-2}^{C_{N_{d}}} & P_{N_{d}-1, N_{d}-1}^{C_{N_{d}}} & 0 \\
0 & \cdots & 0 & P_{N_{d}, N_{d}-M}^{C_{N_{d}}} & P_{N_{d}, N_{d}-M+1}^{C_{N_{N}}} & \cdots & P_{N_{d}, N_{d}-1}^{C_{N_{d}}} & P_{N_{d}, N_{d}}^{C_{N_{d}}}
\end{array}\right], \\
& P_{j, j}^{C_{N_{d}}}= \begin{cases}q * p_{1}, & j=0,1, \ldots, N_{e}-1 \\
0, & j=N_{e},\end{cases} \\
& P_{j, j-m}^{C_{N_{d}}}=\left\{\begin{array}{ll}
(1-q) * p_{m}+q * p_{m+1}, & j=m, m+1, \ldots, N_{e}-1 \\
p_{m}, & j=N_{e},
\end{array} \quad m=1,2, \ldots, M-1,\right. \\
& P_{j, j-m}^{C_{N_{d}}}= \begin{cases}(1-q) * p_{m}, & j=m, m+1, \ldots, N_{e}-1 \\
p_{m}, & j=N_{e},\end{cases}
\end{aligned}
$$




\section{Performance Analysis for CPQM}

We define the steady state distribution (SSD) of the CQPM as $\pi=(\pi(i, j)), i=0,1, \ldots, N_{d}, j=0,1, \ldots, N_{e}$, which represent the probability of each state when system is stable or to say the time shaft is infinity. For the convenience of performance analysis, the steady state distribution of data queue length and energy queue length are defined as follows:

$$
\begin{aligned}
\pi_{d} & =\left(\pi_{d}(i)\right), \quad i=0,1, \ldots, N_{d}, \\
\pi_{d}(i) & =\sum_{j=0}^{N_{e}} \pi(i, j), \\
\pi_{e} & =\left(\pi_{e}(j)\right), \quad j=0,1, \ldots, N_{e}, \\
\pi_{e}(j) & =\sum_{i=0}^{N_{d}} \pi(i, j),
\end{aligned}
$$

where $\pi_{d}$ and $\pi_{e}$ are the steady state distribution of data queue length and energy queue length, respectively. Applying the classic queuing theory [17] and matrix of transition probability which is deduced previously, we can achieve the SSD of the CQPM through the equation set as follows:

$$
\begin{aligned}
\boldsymbol{\pi} & =\boldsymbol{\pi} \mathbf{P}, \\
\sum_{i=0}^{N_{d}} \sum_{j=0}^{N_{e}} \pi(i, j) & =1 .
\end{aligned}
$$

In order to obtain the SSD of the CQPM quickly, we usually set the initial value of $\boldsymbol{\pi}=(1,0,0, \ldots, 0)$. Then, the average length of the data queue and energy queue can be acquired as

$$
\begin{gathered}
\text { Queue }_{\text {data }}=\sum_{i=0}^{N_{d}} i \pi_{d}(i)=\sum_{i=0}^{N_{d}} i\left(\sum_{j=0}^{N_{e}} \pi(i, j)\right), \\
\text { Queue }_{\text {energy }}=\sum_{j=0}^{N_{e}} j \pi_{e}(j)=\sum_{j=0}^{N_{e}} j\left(\sum_{i=0}^{N_{d}} \pi(i, j)\right) .
\end{gathered}
$$

After much effort, we can deduce some performance parameters such as average throughput, average delay, and average drop rate for the CQPM by using formula (25). The average throughput of the data queue and energy queue can be acquired as

$$
\begin{aligned}
\text { Throughput }_{\mathrm{data}} & =\left(1-\pi_{d}(0)\right)\left(\sum_{i=1}^{M} P_{i} \sum_{j=i}^{N_{e}} \pi_{e}(j)\right), \\
\text { Throughput }_{\text {energy }} & =\left(1-\pi_{d}(0)\right)\left(\sum_{i=1}^{M} i P_{i} \sum_{j=i}^{N_{e}} \pi_{e}(j)\right) .
\end{aligned}
$$

The average delay of the data queue and energy queue can be acquired as

$$
\begin{aligned}
\text { Delay }_{\text {data }} & =\frac{\text { Queue }_{\text {data }}}{\text { Throughput }_{\text {data }}} \\
& =\frac{\sum_{i=0}^{N_{d}} i\left(\sum_{j=0}^{N_{e}} \pi(i, j)\right)}{\left(1-\pi_{d}(0)\right)\left(\sum_{i=1}^{M} P_{i} \sum_{j=i}^{N_{e}} \pi_{e}(j)\right)}, \\
\text { Delay }_{\text {energy }} & =\frac{\text { Queue }_{\text {energy }}}{\text { Throughput }_{\text {energy }}} \\
& =\frac{\sum_{j=0}^{N_{e}} j\left(\sum_{i=0}^{N_{d}} \pi(i, j)\right)}{\left(1-\pi_{d}(0)\right)\left(\sum_{i=1}^{M} i P_{i} \sum_{j=i}^{N_{e}} \pi_{e}(j)\right)} .
\end{aligned}
$$

The average drop rate of the data queue and energy queue can be acquired as

$$
\begin{gathered}
\operatorname{Drop}_{\text {data }}=\pi_{d}\left(N_{d}\right)=\sum_{j=0}^{N_{e}} \pi\left(N_{d}, j\right), \\
\text { Dropenergy }_{\text {en }}=\pi_{e}\left(N_{e}\right)=\sum_{i=0}^{N_{d}} \pi\left(i, N_{e}\right) .
\end{gathered}
$$

\section{Simulation and Analysis Results}

We have deduced the performances of average queue length, average throughput, average delay, and average drop rate about both data packet and energy packet for the CQPM in the previous sections. In this section, we will compare the deduced performances of the CQPM with the performances profile which is obtained by Monte-Carlo method and then analyze the physical significance of them which may provide a guide for the real system. Without loss of generality, the buffer sizes of data queue are $N_{d}=8$ and the buffer sizes of energy queue are $N_{e}=12$. The curve with "CPT $M=1$ " represents that the relay adopts $\mathrm{CPT}$ strategy which acts as a benchmark to show the performance gain of taking the APT strategy into consideration. The curves with "APT $M=2,3,4$ " represent that the relay adopts APT strategy which allows sacrificing more energy packets to gain a better system performance in the bad channel state case due to sufficient energy. The channel condition dividing value is set as $M=1,2,3,4$. For Bernoulli arrival process, the data arrival rate parameter $p$ and energy arrival rate parameter $q$ satisfy $0 \leq p \leq 1$ and $0 \leq q \leq 1$, respectively.

Seen from Figure 2, the theoretical value and the computer simulation value are consistent which indicates the validity of the theoretical derivation. Then, we analyze the performance of the average data queue length and energy queue length when $M=1,2,3,4$ in the $C P Q M$ which are profiled in Figure 2. Compared with traditional CPT strategy, the proposed new relay protocol with APT strategy has more advantage. Actually, the CPT strategy is equivalent to the APT strategy with $M=1$. Figure 2(a) shows the relationship between average data queue length and average data arrival rate with a constant average energy arrival rate $q=0.8$. 


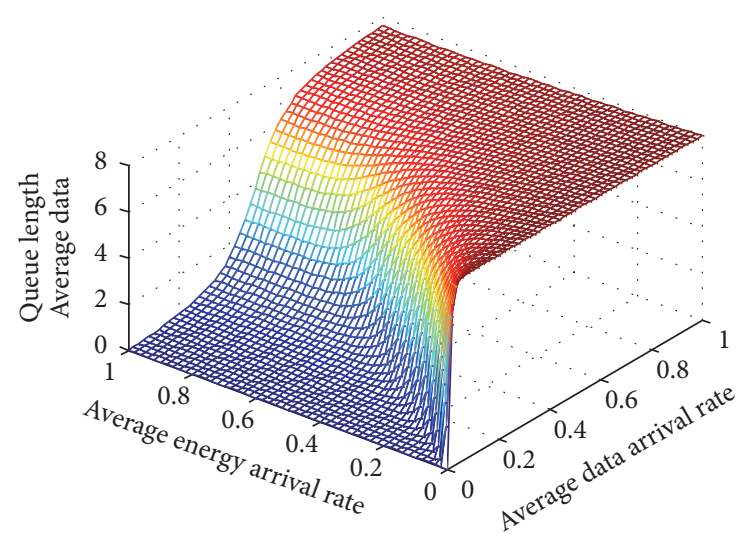

(a) Average data queue length

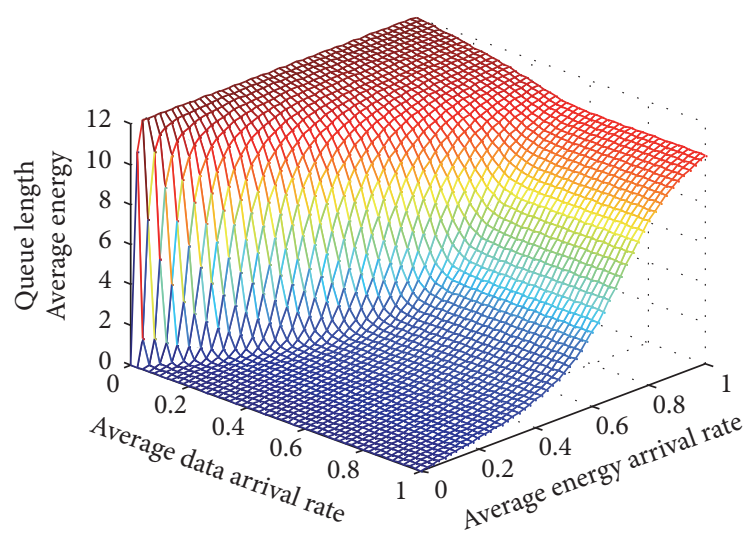

(b) Average energy queue length

FIGURE 3: The average queue length performance of CPQM with average data arrival and energy arrival when $M=2$.

No matter what we set the $M$ value, the average data queue length starts to increase and then saturates with the increase of average data arrival rate. However, the larger $M$ value which means that we will sacrifice more energy packets to send one data packet does not always achieve a better system performance. We can see from Figure 2(a) that the larger $M$ value will obtain a smaller average data queue which means the data will be sent quickly with less delay when the average data arrival rate satisfies $p \leq 0.34$. Once the average data arrival rate satisfies $p>0.34$, we will find that the larger $M$ value may not be the best choice, because when the average data arrival rate $p$ gets larger this actually means that there are more data packets that need be sent and the existing energy packet may not be abundant. Specially in Figure 2(a), $M=3$ has the best performance than the others when the average data arrival rate satisfies $0.34 \leq p \leq 0.52$, but $M=2$ will be the best choice when $p \geq 0.58$.

Figure 2(b) shows the relationship between average data queue length and average energy arrival rate with a constant average data arrival rate $p=0.3$. The average data queue length starts to decrease and then saturates with the increase of average energy arrival rate regardless of the different $M$ values. Viewed from another perspective, the smallest data queue length will have the best performance index because a large proportion of data packets have been sent out. We can also sacrifice more energy packets to send one data packet which means setting the larger $M$ value, but the best $M$ value will change with the increase of the average energy arrival rate. As seen in Figure 2(b), $M=1$ has better performance than the others when the average energy arrival rate satisfies $q \leq 0.35$. It is because sacrificing more energy packets to send one data packet will lead to no energy for sending the remaining data packets when the average energy arrival rate is very small. Once the average energy arrival rate gets larger, it make sense to sacrifice more energy packets to send one data packet in the bad channel state case due to sufficient energy. Specially in Figure 2(b), $M=1$ has better performance than the others when the average energy arrival rate satisfies $q \leq$ 0.35 ; then $M=2$ will be the best choice when $0.35 \leq q \leq 0.56$. Afterwards with the increase of the average energy arrival rate, more larger $M$ values will contribute to improve the system performance. When the average energy arrival rate $0.56 \leq q \leq 0.74, M=3$ has better performance than the others. Once the average energy arrival rate $q \geq 0.74$, we will set $M=4$ to achieve a better performance.

Figure 3 illustrates the average queue length performance of CPQM with average data arrival and energy arrival when $M=2$. Showed in Figure 3(a), the average data queue length starts to grow and then saturates with the increase of the average data arrival rate and energy arrival rate. The Queue $_{\text {data }}$ achieve saturation rapidly with the increase of the average data arrival rate when the $q$ is very small. With the same condition, if the average energy arrival rate gets larger, the Queue $_{\text {data }}$ will increase gently and then be saturated. On the contrary in Figure 3(b), the average energy queue length Queue $_{\text {energy }}$ starts to decrease and then saturates with the increase of the average data arrival rate and energy arrival

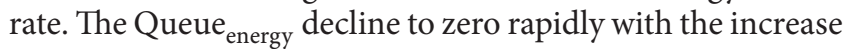
of the average data arrival rate when $q$ is very small because the vast data arrival exhausts the limited energy arrival. When the average energy arrival rate gets larger, the Queue $_{\text {data }}$ will decrease gently and then be saturated at a nonzero value which indicates that the energy is enough to send all the data packets that arrived.

Also shown in Figure 4, the theoretical value and the computer simulation value are consistent which indicates the validity of the theoretical derivation. Figure 4 illustrates that the average data throughput of CPQM starts to increase and then saturates with the increase of the average data arrival rate or energy arrival rate when $M=1,2,3,4$. As mentioned previously, adopting larger $M$ value and expending more energy packets to send one data packet when channel state is bad may decrease the data delay in the buffer but did not always achieve the best data throughput performance. Shown in Figure 4(a), $M=2$ is the best choice with the increase of the average data arrival when $q=0.5$. In another case, Figure 4(b) displays that the average data throughput of $\mathrm{CPQM}$ starts to increase and then saturates with the increase of the average energy arrival and $p=0.5$. When $q \leq 0.37$ the strategy $M=1$ will have better date throughput than the 


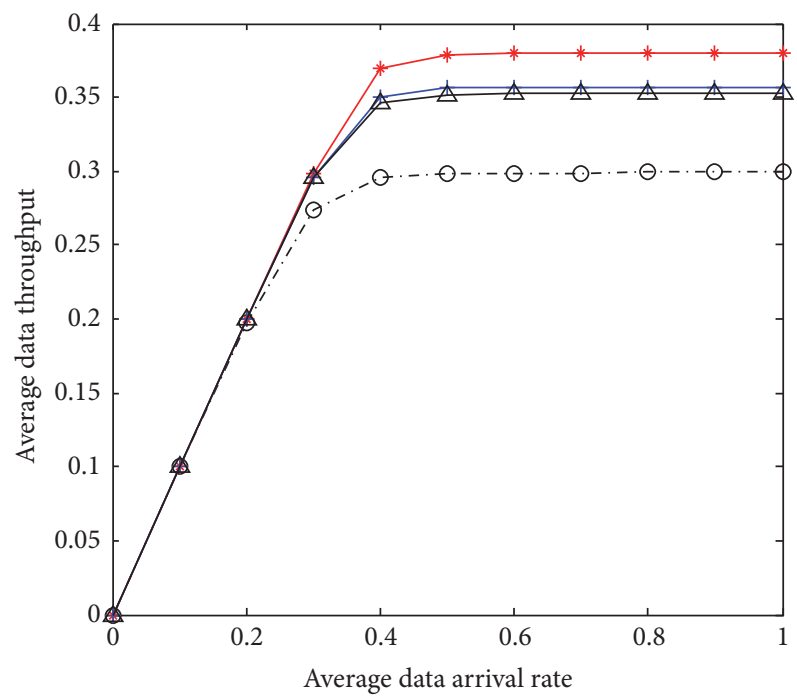

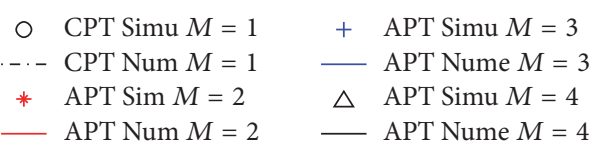

(a) Average data throughput with $q=0.5$

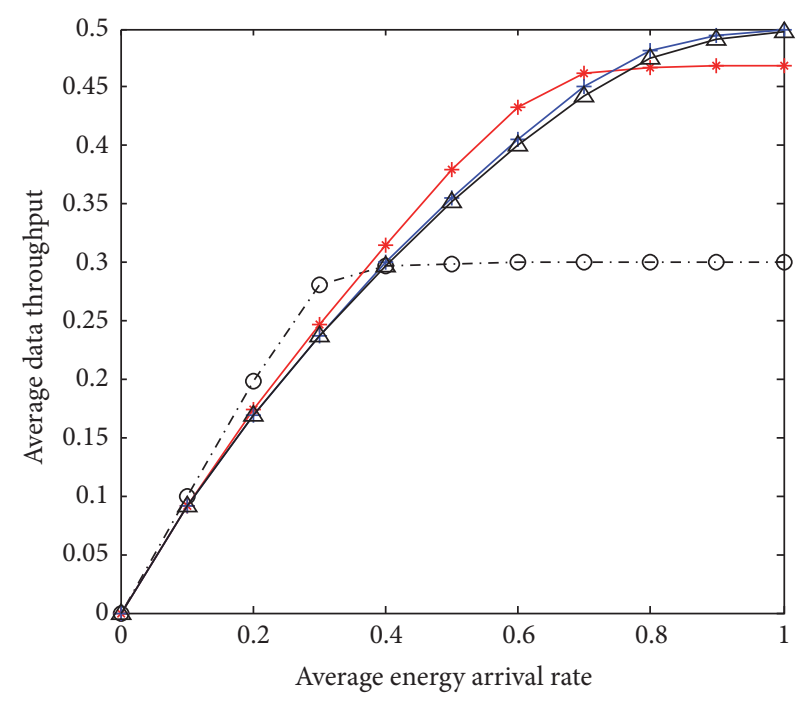

- $\quad$ CPT Simu $M=1 \quad+\quad$ APT Simu $M=3$

..- CPT Num $M=1 \quad$ APT Nume $M=3$

* $\quad$ APT $\operatorname{Sim} M=2 \quad \triangle \quad$ APT Simu $M=4$

— APT Num $M=2$ - APT Nume $M=4$

(b) Average data throughput with $p=0.5$

FIGURE 4: The average data throughput performance of CPQM with average data arrival and $M=1,2,3,4$.

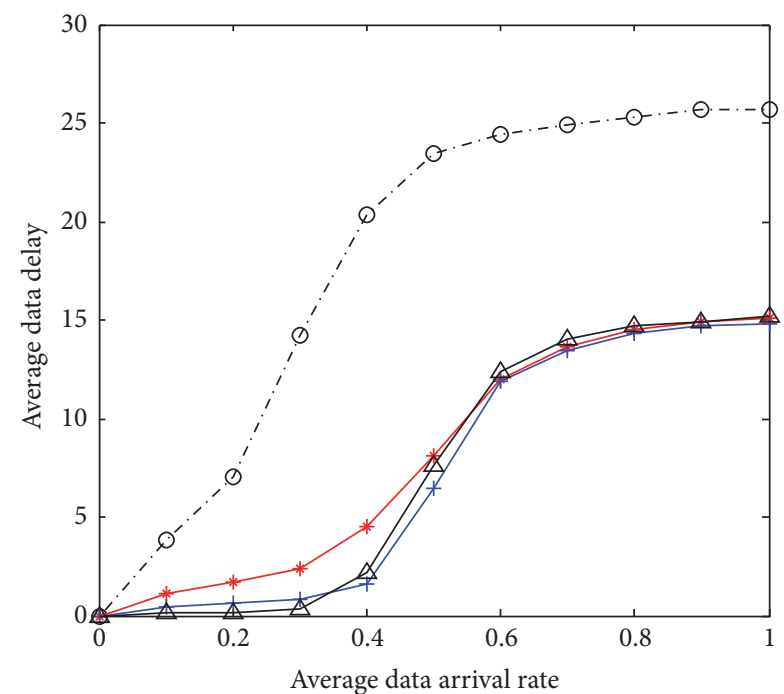

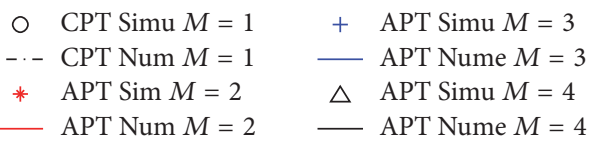

(a) Average data delay with $q=0.8$

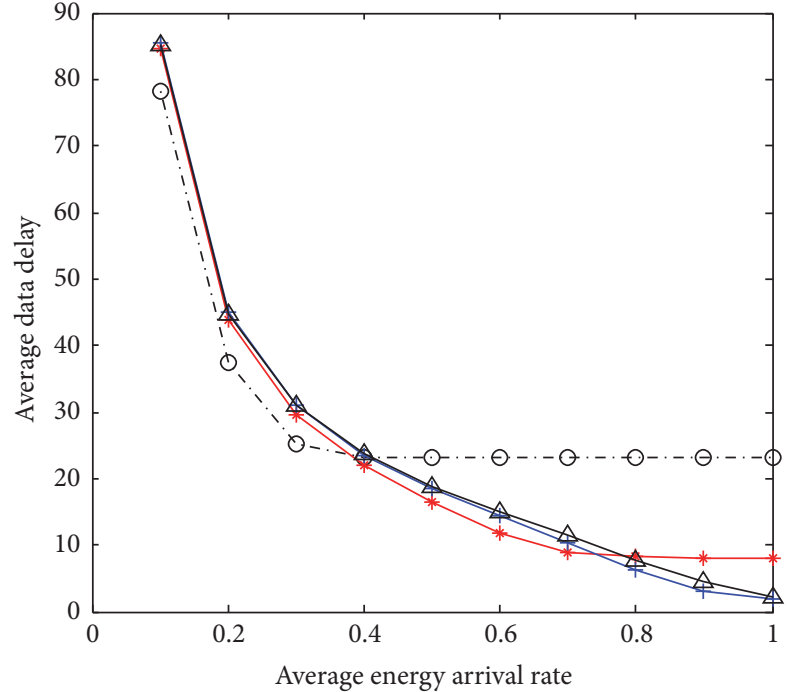

○ $\quad$ CPT Simu $M=1 \quad+\quad$ APT Simu $M=3$

- - CPT Num $M=1 \quad$ APT Nume $M=3$

* APT $\operatorname{Sim} M=2 \quad \triangle \quad$ APT Simu $M=4$

- APT Num $M=2 \quad$ APT Nume $M=4$

(b) Average data delay with $p=0.5$

FIGURE 5: The average data delay performance of CPQM with $M=1,2,3,4$.

others due to poor energy arrival rate. With the increase of the energy arrival rate, the choice $M=2$ will have better date throughput than the others when $0.37 \leq q \leq 0.76$ and the strategy $M=3$ will be the best choice when $q \geq 0.76$. Note that if we set more large data arrival rate, the choice $M=4$ may also be the best choice if there is plenty of energy to use.
We see from Figure 5, the theoretical value and the computer simulation value are consistent which indicates the validity of the theoretical derivation. Figure 5 illustrates that the average data delay of CPQM starts to increase and then saturates with the increase of the average data arrival and different energy arrival rate when $M=1,2,3,4$. As seen from 


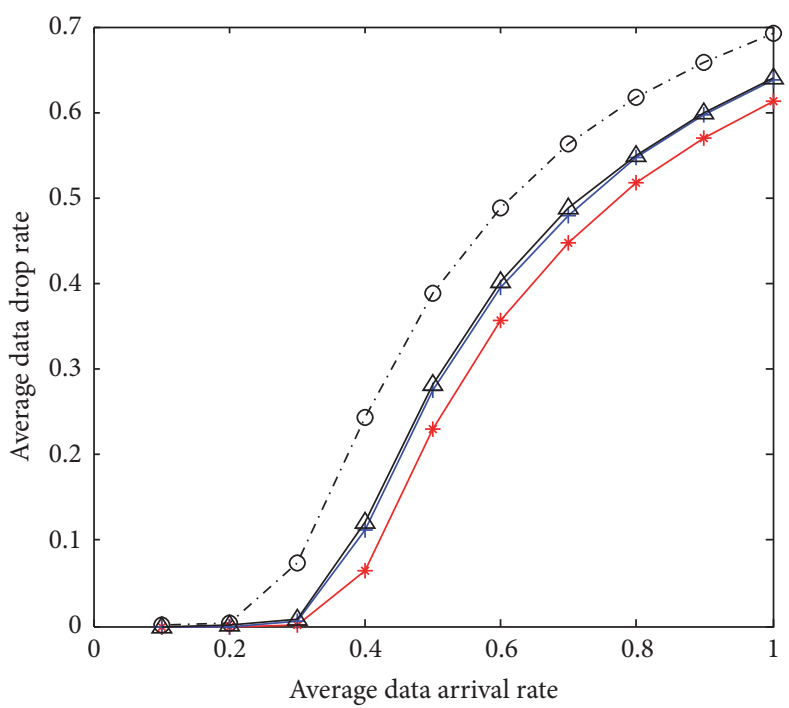

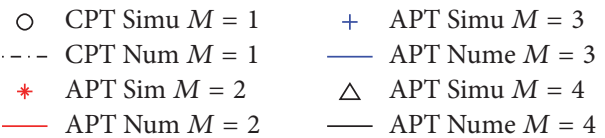

(a) Average data drop rate with $q=0.5$

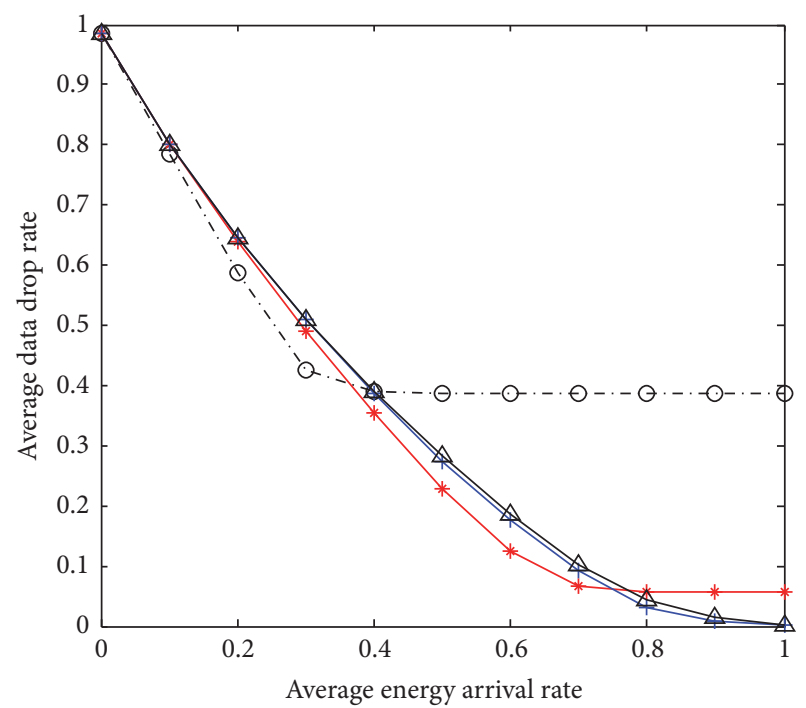

○ CPT Simu $M=1 \quad+$ APT Simu $M=3$

-. CPT Num $M=1 \quad$ APT Nume $M=3$

* $\quad$ APT $\operatorname{Sim} M=2 \quad \triangle$ APT Simu $M=4$

— APT Num $M=2 \quad$ APT Nume $M=4$

(b) Average data drop rate with $p=0.5$

FIGURE 6: The average data drop rate performance of CPQM with $M=1,2,3,4$.

the figures, adopting larger $M$ value and expending more energy packets to send one data packet when channel state is bad did not always obtain the lowest data delay performance. It is because the luxuriant energy expend strategy may generate that the remaining data has no energy for sending which instead increases the data delay. Specially in Figure 5(a) with energy arrival rate $q=0.8$, the strategy $M=2,3,4$ has huge delay performance gain compared with $M=1$ if we have overmuch energy to exhaust. When the data arrival rate is less than energy arrival rate relatively such as $p \leq 0.34$ and $q=0.8$, then $M=4$ is the best choice due to sufficient energy. Once the data arrival rate is comparable with energy arrival rate such as $p \geq 0.34$ and $q=0.8$, we may not have enough energy to overspend, so the largest $M$ value could not achieve the best delay performance. As seen from Figure 5(a), $M=3$ has smaller delay than the others when $p \geq 0.34$ and $q=0.8$. Figure 5(b) displays that the average data delay starts to decrease and then remains unchanged with the increase of the average energy arrival and data arrival $p=0.5$ no matter what we set the $M$ value. $M=1$ has smaller delay than the others when average energy arrival $q \leq 0.37$. When the energy arrival rate gets larger, more larger $M$ values will achieve the best delay performance more easily. As shown in Figure 5(b), $M=2$ is the best choice when $0.37 \leq q \leq 0.76$, but $M=3$ will have the best delay performance when average energy arrival $q \geq 0.76$.

Also shown in Figure 6, the theoretical value and the computer simulation value are consistent which indicates the validity of the theoretical derivation. Figure 6 display the average data drop rate and energy drop rate of CPQM when $M=2$. As shown in Figure 6(a), the average data drop rate increases with the increase of the average data arrival and energy arrival $q=0.5$ no matter what we set the $M$ value. When the data arrival rate is very small the data drop rate is zero for all the strategies with a constant energy arrival rate because all the data can be sent. The $M=1$ strategy starts to increase early than the others when $p \geq 0.2 . M=$ 2,3,4 have less data drop rate than $M=1$ due to more flexible transmission protocol although it takes more energy to expend. As we mentioned before, the biggest $M$ value may not be the best choice unless we have energy to exhaust. As seen from Figure 6(a), the $M=2$ strategy has better data overflow performance when $p \geq 0.2$ and $q=0.5$. On the contrary in Figure 6(b), the average data drop rate starts to decrease and then holds still with the increase of energy arrival and a constant data arrival. $M=1$ has smaller data drop rate performance than the others when average energy arrival $q \leq 0.37$ because of insufficient energy. Note that with the energy arrival rate getting bigger, more larger $M$ values will achieve a better system performance more easily. Specially, $M=2$ is the best choice when $0.37 \leq q \leq 0.76$, but $M=3$ will have the best data drop rate when average energy arrival $q \geq 0.76$.

According to Figures 2-6, it seems that better results are obtained when we adopt APT strategy if there is abundant energy arrival. It would be interesting to collect or fix the optimal value of $M$ in order to obtain better results in terms of delay, throughput, and data drop. More detail can be seen from Figure 7 when we consider the system performance with fixed $M=1,2,3,4$. In the scenario of Case A with $p=$ $0.5, q=0.3$, where the average energy arrival rate is less than the average data arrival rate, the CPT strategy with $M=1$ achieves a higher data throughput and a lower data delay and drop rate due to poor energy arrival. With the increase of 

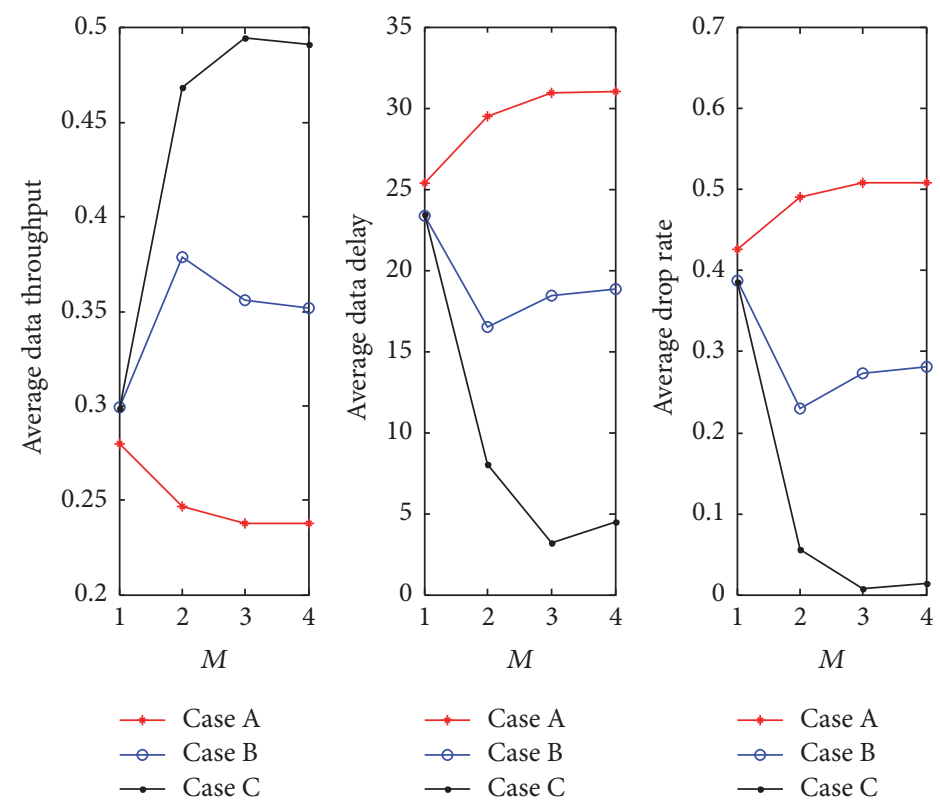

Figure 7: The average system performance of CPQM with fixed $M=1,2,3,4$. Case A: $p=0.5, q=0.3$. Case B: $p=0.5, q=0.5$. Case C: $p=0.5, q=0.9$.

the $M$ value, the system performance decreases due to the insufficient energy packet. In Case $\mathrm{B}$ with $p=0.5, q=$ 0.5 , where the average energy arrival rate is equal to the average data arrival rate, the system performance improves and then deteriorates with the increase of the $M$ value. As seen from the figure, adopting APT strategy with $M=2$ is the best choice. Once the energy arrival is abundant compared with data arrival, the APT with larger $M$ value will obtain more performance advantage. Specially in Case $\mathrm{C}$ with $p=$ $0.5, q=0.9$, where the average energy arrival rate is more abundant than the average data arrival rate, the APT strategy with $M=3$ obtains the best system performance. As we mentioned before, the APT strategy with the biggest $M$ value may not be the best choice unless we have energy to exhaust.

\section{Conclusion}

This paper focuses on the performance analysis of joint data packet and energy packet handling model for cooperative relaying networks. After describing the relaying system model, a new relay transmit protocol which considers the influence of channel condition on the data departure rate is proposed. The data arrival and energy arrival coupling queuing problem is ascribed to a Coupled Processor Queuing Model. Applying Quasi-Birth and Death method, the steady state transition probability of the data and energy coupled model is obtained and the expressions of relaying system performance parameters are also derived. Simulations are demonstrated to verify the analytical results under different data arrival rate, energy arrival rate, and relaying strategy. In particular, a better throughput/delay-energy expedition tradeoff is obtained when channel condition is bad. But the APT strategy with the biggest $M$ value may not be the best choice, because the luxuriant energy expend strategy may generate that the remaining data has no energy for sending.

\section{Conflicts of Interest}

The authors declare that they have no conflicts of interest.

\section{Acknowledgments}

The work is supported by Natural Science Foundation of China (61571234, 61401225), National Program on Key Basic Research Project (no. 2013CB329005), Jiangsu Provincial National Science Foundation (BK20140894), the Key Projects of Support Program for Outstanding Youth Talent of Universities in Anhui Province (gxyqZD2016123), and Youth Foundation of Anhui Polytechnic University (2012YQ39, 2014YQ40).

\section{References}

[1] J. N. Laneman, D. N. C. Tse, and G. W. Wornell, "Cooperative diversity in wireless networks: efficient protocols and outage behavior," IEEE Transactions on Information Theory, vol. 50, no. 12, pp. 3062-3080, 2004.

[2] R. U. Nabar, H. Bölcskei, and F. W. Kneubühler, "Fading relay channels: performance limits and space-time signal design," IEEE Journal on Selected Areas in Communications, vol. 22, no. 6, pp. 1099-1109, 2004.

[3] G. Kramer, M. Gastpar, and P. Gupta, "Cooperative strategies and capacity theorems for relay networks," IEEE Transactions on Information Theory, vol. 51, no. 9, pp. 3037-3063, 2005.

[4] N. Zlatanov, A. Ikhlef, T. Islam, and R. Schober, "Buffer-aided cooperative communications: opportunities and challenges," 
IEEE Communications Magazine, vol. 52, no. 4, pp. 146-153, 2014.

[5] M. Shaqfeh, A. Zafar, H. Alnuweiri, and M. Alouini, "Maximizing expected achievable rates for block-fading buffer-aided relay channels," IEEE Transactions on Wireless Communications, vol. 15, no. 9, pp. 5919-5931, 2016.

[6] Z. Tian, Y. Gong, G. Chen, and J. A. Chambers, "Bufferaided relay selection with reduced packet delay in cooperative networks," IEEE Transactions on Vehicular Technology, vol. 66, no. 3, pp. 2567-2575, 2017.

[7] X. Jia, P. Deng, D. Wang, L. Yang, and H. Zhu, "Outage performance analysis for buffer-aided relay system over nonidentical Rayleigh fading channels," IET Communications, vol. 9, no. 15, pp. 1842-1851, 2015.

[8] B. Zhang, C. Dong, J. Lei, M. El-Hajjar, L.-L. Yang, and L. Hanzo, "Buffer-aided relaying for the multi-user uplink: outage analysis and power allocation," IET Communications, vol. 10, no. 8, pp. 936-944, 2016.

[9] S. Priya and D. J. Inman, Energy Harvesting Technologies, Springer, New York, NY, USA, 2009.

[10] J. Yang and S. Ulukus, "Optimal packet scheduling in an energy harvesting communication system," IEEE Transactions on Communications, vol. 60, no. 1, pp. 220-230, 2012.

[11] C. Huang, R. Zhang, and S. Cui, "Throughput maximization for the gaussian relay channel with energy harvesting constraints," IEEE Journal on Selected Areas in Communications, vol. 31, no. 8, pp. 1469-1479, 2013.

[12] B. Varan and A. Yener, "Delay constrained energy harvesting networks with limited energy and data storage," IEEE Journal on Selected Areas in Communications, vol. 34, no. 5, pp. 15501564, 2016.

[13] S. Gupta, R. Zhang, and L. Hanzo, “Throughput maximization for a buffer-aided successive relaying network employing energy harvesting," IEEE Transactions on Vehicular Technology, vol. 65, no. 8, pp. 6758-6765, 2016.

[14] V. Hakami and M. Dehghan, "Distributed power control for delay optimization in energy harvesting cooperative relay networks," IEEE Transactions on Vehicular Technology, 2016.

[15] H. Ju and R. Zhang, "Throughput maximization in wireless powered communication networks," IEEE Transactions on Wireless Communications, vol. 13, no. 1, pp. 418-428, 2014.

[16] S. Luo, G. Yang, and K. C. Teh, "Throughput of wirelesspowered relaying systems with buffer-aided hybrid relay," IEEE Transactions on Wireless Communications, vol. 15, no. 7, pp. 4790-4801, 2016.

[17] D. Gross and C. M. Harris, "Fundamentals of queuing theory," Mathematical Gazette, 1998.

[18] L. Lei, Y. Zhang, X. S. Shen, C. Lin, and Z. Zhong, "Performance analysis of device-to-device communications with dynamic interference using stochastic petri nets," IEEE Transactions on Wireless Communications, vol. 12, no. 12, pp. 6121-6141, 2013.

[19] A. Tandon and M. Motani, "Has green energy arrived? Delay analysis for energy harvesting communication systems," in Proceedings of the 11th Annual IEEE International Conference on Sensing, Communication, and Networking (SECON'14), pp. 582590, IEEE, Singapore, July 2014.

[20] J. S. H. Van Leeuwaarden and J. A. C. Resing, "A tandem queue with coupled processors: computational issues," Queueing Systems, vol. 51, no. 1-2, pp. 29-52, 2005. 


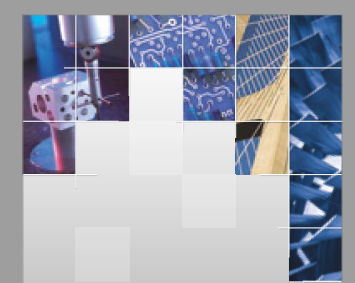

\section{Enfincering}
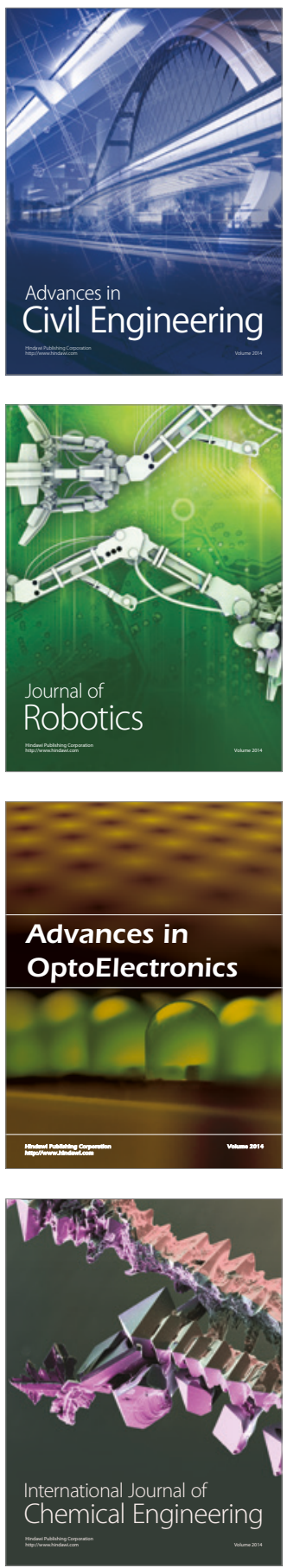

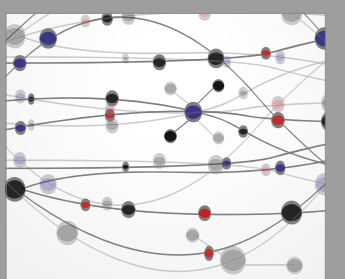

The Scientific World Journal

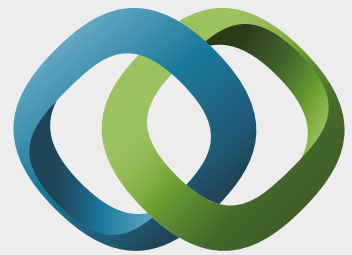

\section{Hindawi}

Submit your manuscripts at

https://www.hindawi.com
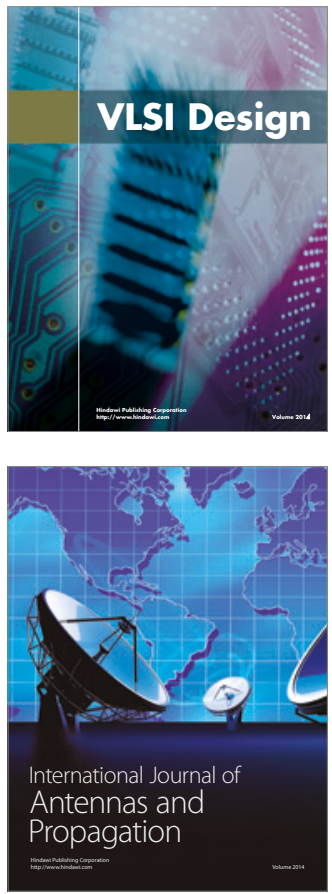

\section{Rotating}

Machinery
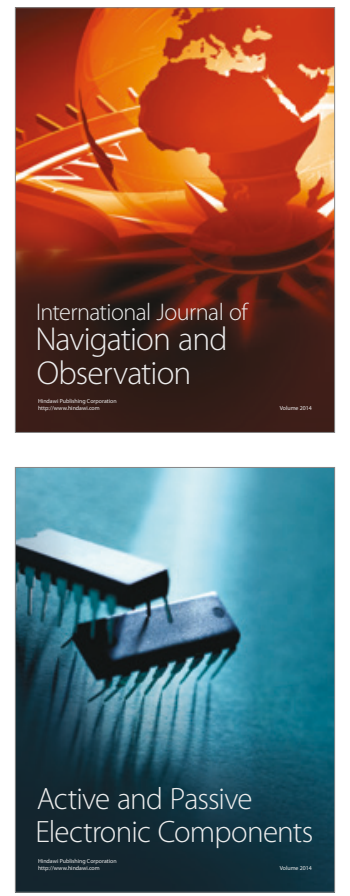
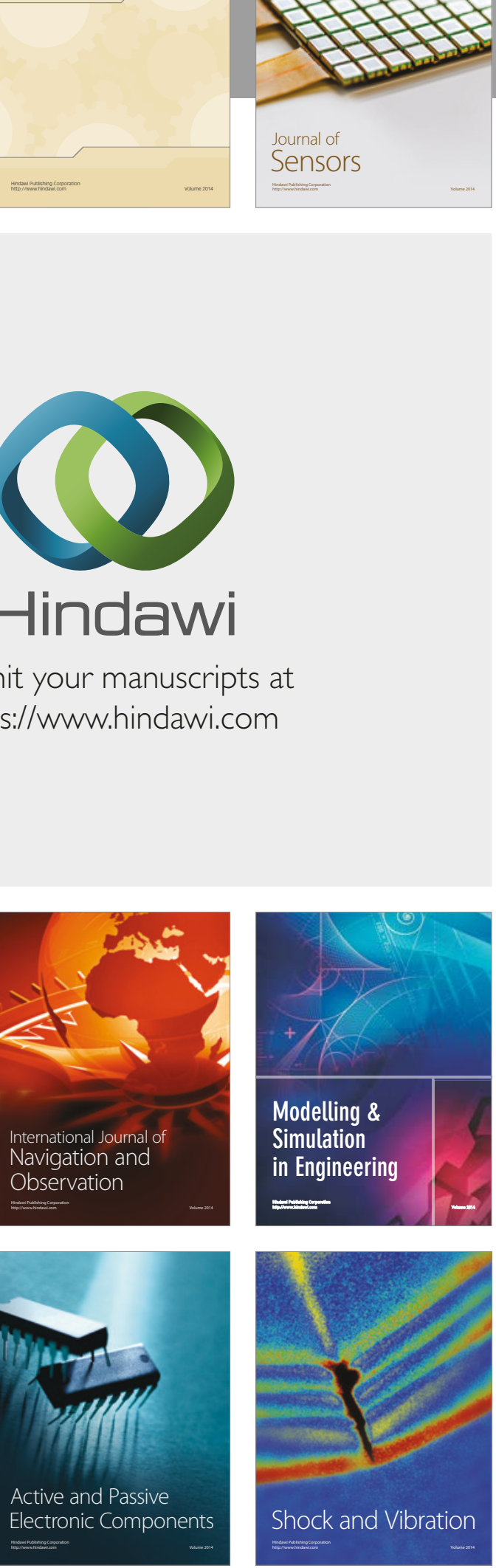
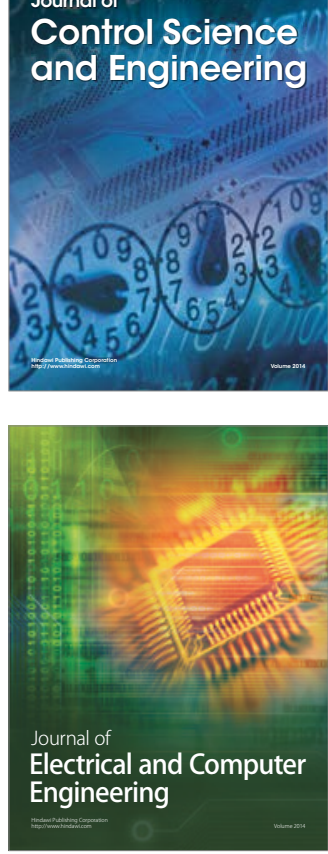

Distributed

Journal of

Control Science

and Engineering
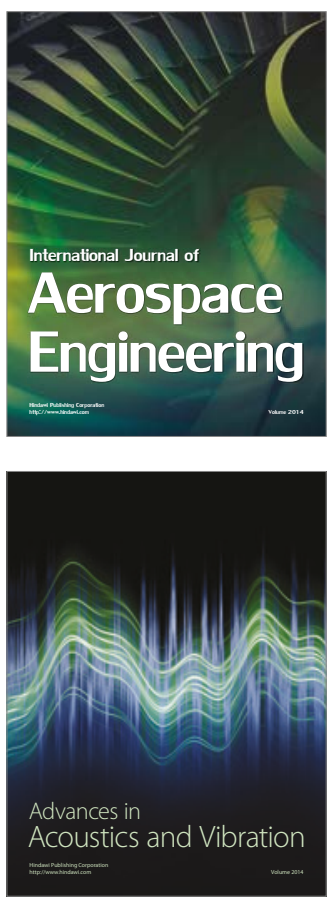

Sensor Networks 